\title{
Visualization of Subjective Evaluation for the Participation Attitude to the Internet of Male College Students Based on Visual Analog Scale
}

\author{
Satoshi Watanabe ${ }^{\mathrm{a}, *}$, Toshiro Tsuruta ${ }^{\mathrm{a}}$, Naruki Shirahama ${ }^{\mathrm{b}}$, Naofumi Nakaya ${ }^{\mathrm{c}}$, \\ Yuji Matsumoto ${ }^{\mathrm{d}}$, Hiroyuki Tsukamoto ${ }^{\mathrm{e}}$ and Yukio Mori ${ }^{\mathrm{f}}$

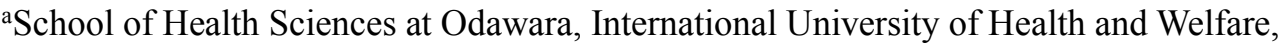 \\ 1-2-25, Shiroyama, Odawara-shi, Kanagawa, 250-8588, Japan \\ ${ }^{b}$ Department of Creative Engineering, National Institute of Technology, Kitakyushu College, \\ 5-20-1, Shii, Kokuraminami-ku, Kitakyushu-shi, Fukuoka, 802-0985, Japan \\ 'Faculty of Health Sciences, Tsukuba International University, 6-20-1, Manabe, Tsuchiura-shi, Ibaraki, 300-0051, Japan \\ dJunior College Division, Kindai University, 3-4-1, Kowakae, Higashiosaka-shi, Osaka, 577-8502, Japan \\ eSchool of Information Studies, Shizuoka Sangyo University, \\ 4-1-1, Surugadai, Fujieda-shi, Shizuoka, 426-8668, Japan \\ fDepartment of Mechanical and Electronic Engineering, Salesian Polytechnic, \\ 4-6-8, Oyamagaoka, Machida-shi, Tokyo, 194-0215, Japan \\ *Corresponding Author: satoshi-w@iuhw.ac.jp
}

\begin{abstract}
This paper describes the visualizations of subjective evaluation for the participation attitude to the Internet of male college students based on visual analog scale (VAS) by employing a small number of subjective evaluation measurement.

19 healthy male college students were invited as the experiment participants. In order to analyze their subjective evaluation for the participation attitude to the Internet, they were requested to answer the questionnaire on VAS (17 questions). The results showing that values based on their subjective evaluation ("applicable" or "not applicable ") for the participation attitude to the Internet have been obtained, and they are shown by using the combination of univariate scatter plots and box-and-whisker plots.

Moreover, it has been discovered that the question based on the similar subjective evaluation belongs to the same cluster. This fact is supported by the results of published article ${ }^{(12)}$ analyzed in factor analysis.

Under above procedure, relationships and meanings for these questions have been found by a small number of subjective evaluation measurement based on VAS.
\end{abstract}

Keywords: subjective evaluation, visual analog scale, combination of univariate scatter plot and box-and-whisker plot.

\section{Introduction}

Studies on the subjective evaluation based on Likert Scale (LS, see Fig.1 (a)) ${ }^{(1)}$ have been conducted broadly. LS is a simple discrete scale method, but it has some weak points as follows ${ }^{(1-5)}$ : (1) LS's evaluation often becomes complicated; (2) Sometimes several biases are introduced by the following factors, such as halo effect, leniency effect, and central tendency; and (3) LS's results are discrete data.

From these points, it is suggested that there exists unintended bias on LS's results. In order to solve these weak points, Visual Analog Scale (VAS, see Fig.1(b)) is investigated on the subjective evaluation ${ }^{(6,7)}$. VAS is a ratio scale method, it is expected that it can solve the weak points of LS as mentioned above ${ }^{(6,7)}$. And it is considered that VAS is more flexible than LS.

Therefore, we investigated on the trend of the subjective evaluation based on VAS and LS, existence of unintended bias between LS data and VAS data was discovered $^{(6,7)}$.

Moreover, we also investigated on the subjective evaluation based on VAS, such as impression of music ${ }^{(8,9)}$, impression received from the response of a communication robot $^{(10)}$, and so on. From these investigations, it could be 
able to get the results that are the same with our feelings ${ }^{(8-10)}$.

On the other hand, in general, it is necessary to lots of measurements of subjective evaluation based on LS to obtain reliable conclusions.

However, as a health consultation activity, it needs to give an accurate result by a small number of subjective evaluation measurement. To understand these data's distribution and other characteristics, Weissgerber et al. proposes that combination of univariate scatter plot and box-and-whisker plot of them should be drawn ${ }^{(11)}$. Therefore, this proposed $\operatorname{method}^{(11)}$ is applied for our investigations $^{(8-10)}$.

That is, as same as our previous studies ${ }^{(8-10)}$, to visualize the experimental results, combination of univariate scatter plot and box-and-whisker plot $^{(11)}$ are also used in this paper.

Under such conditions, visualization analysis method with a small number of subjective evaluation measurement based on VAS have been proposed ${ }^{(8-10)}$. This method consists of following components: (1) Subjective evaluation measurements based on VAS (values are 0.00-1.00); (2) Calculation of basic statistics; (3) Calculation of a correlation matrix; (4) Drawing of combination of univariate scatter plot and box-and-whisker plot ${ }^{(11)}$; (5) Hierarchical clustering analysis by Ward method; and (6) Non-hierarchical clustering analysis by k-means.

In this method, relationships and meanings of subjective evaluation values can be analyzed. This fact is consistent with our senses and it is evidence that can be analyzed by a small number of subjective evaluation measurement based on $\operatorname{VAS}^{(8-10)}$.

Under the above preparation, this paper describes the visualizations of subjective evaluation for the participation attitude to the Internet of male college students based on VAS by a small number.

The remainder of this paper is organized as follows. Section 2 describes the theory of VAS and its visualization analysis. Materials and methods are shown in Section 3. Results and discussion are given in Section 4. Section 5 shows the conclusions and future works.

\section{Theory of Subjective Evaluation Based on VAS and its Visualization}

\subsection{VAS}

Recently, VAS has been applied to study on the subjective evaluation such as education ${ }^{(6,7,10)}$, impression of music $^{(8,9)}$ and so on.

This paper first introduces VAS. Fig.1 (b) shows typical types of VAS's questionnaire. This is to ask the participation attitude to the Internet. To answer Fig. 1 (b), the response is marked by drawing a vertical line on the horizontal line. An example is shown in Fig.2.

Calculation of VAS data is shown in Fig.2. VAS's results are determined by the ratio of (b) to (a) (in other words, it is determined by the position of the mark on the line).

As mentioned above, a value of subjective evaluation (between 0.00 to 1.00 ) can be obtained.

\subsection{Visualization of Subjective Evaluation Based on $\operatorname{VAS}^{(8-10,11)}$}

By the visualization of values obtained in 2.1, it is possible to know their characteristics. To clarify their characteristics, the analysis is carried out by $R$ environment.

First, the basic statistics and a correlation matrix are calculated, the outlines of distribution and relationships of subjective evaluation values are obtained. Next, combination of univariate scatter plots and box-and-whisker plots ${ }^{(11)}$ are drawing, this is our unique $\operatorname{method}^{(8-10)}$. Moreover, hierarchical clustering analysis by

Question: I am always thinking about the Internet.

$\begin{array}{lllllll}\text { Applicable } & 1 & 2 & 3 & 4 & 5 & \text { Not applicable }\end{array}$

(a) LS

Question: I am always thinking about the Internet. Applicable Not applicable

(b) VAS

Fig.1. Typical types of LS and VAS

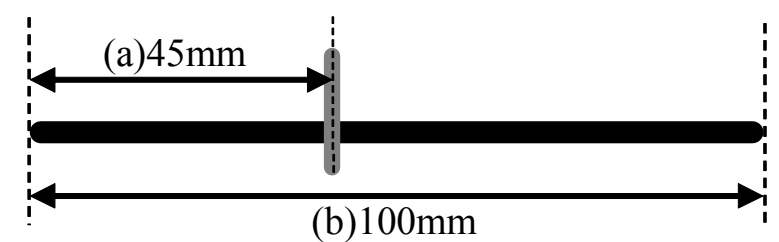

value of subjective evaluation

$$
=(\mathrm{a}) /(\mathrm{b})=0.45(\text { or } 45.0 \%)
$$

Fig.2. Example of calculation of VAS data 
Ward method and non-hierarchical clustering analysis by k-means are carried out.

Under these procedure, relationships and meanings of subjective evaluation values and clustering of participants are understood as the same with our feelings ${ }^{(8-10)}$.

\section{Materials and Methods}

\subsection{Participants}

19 healthy male college students (age: 18.74 \pm 1.21 , mean \pm standard deviation) were invited as the experiment participants, and their basic properties are summarized in Table 1. 11 participants' utilization time of their devices is 4 hours or less, on the other hand, 4 participants' utilization time of their devices is 7 hours or more (For references, 18 participants use their cell-phone of modern models, 6 of them also use another device).

Ethical considerations are as follows:

The explanation of this study was given orally, and the documents were distributed to the participants. The participation in this study was voluntary. Furthermore, the data has been collected anonymously, and has been only used for the presentation of this study.

\subsection{Visualization of Subjective Evaluation Based on VAS}

To analyze their subjective evaluation for the participation attitude to the Internet, they are asked to answer the questionnaire on VAS.

All participants were invited to answer questionnaire relating to the sending and reception of messages on the Internet first (see Table 2, questions from Q1.1 to Q1.7), and then to answer questionnaire relating to the participation attitude to the Internet (see Table 3, questions from Q2.1 to Q2.10). These questionnaires were extracted by Tsuruta et al.'s factor analysis ${ }^{(12)}$, their validity and reliability have been confirmed ${ }^{(12)}$.

Moreover, to clarify their characteristics, the following components are obtained by $\mathrm{R}$ environment as mentioned in 2.2 $2^{(8-10)}$ : (1) Calculation of basic statistics; (2) Calculation of a correlation matrix; (3) Drawing of combination of univariate scatter plot and box-and-whisker plots ${ }^{(11)}$; (4) Hierarchical clustering analysis by Ward method; and (5) Non-hierarchical clustering analysis by k-means.
Table 1. Basic properties of participants

\begin{tabular}{|c|c|c|}
\hline No. & Age & $\begin{array}{c}\text { Utilization time } \\
\text { of participants' } \\
\text { devices* } \\
\text { Time / hour }\end{array}$ \\
\hline 1 & 18 & 6 \\
\hline 2 & 18 & 4 \\
\hline 3 & 20 & 4 \\
\hline 4 & 18 & 2 \\
\hline 5 & 18 & 8 \\
\hline 6 & 19 & 2 \\
\hline 7 & 19 & 3 \\
\hline 8 & 18 & 3 \\
\hline 9 & 19 & 6 \\
\hline 10 & 18 & 11 \\
\hline 11 & 18 & 12 \\
\hline 12 & 18 & 6 \\
\hline 13 & 19 & 3 \\
\hline 14 & 18 & 7 \\
\hline 15 & 18 & 3 \\
\hline 16 & 23 & 3 \\
\hline 17 & 20 & 3 \\
\hline 18 & 18 & 5 \\
\hline 19 & 19 & 3 \\
\hline 1 & $18.74 \pm 1.21^{* *}$ & $4.95 \pm 2.80^{* *}$ \\
\hline
\end{tabular}

All participants are male college student.

*Cell-phone, Personal computer, Tablet terminals, etc.

$* *$ Mean \pm Standard deviation

Table 2. Questionnaires relating to the sending and reception of messages on the Internet for VAS (questions from Q1.1 to Q1.7)

Q1.1: I get worried if I receive no response to my message.

Q1.2: I want to check my cell-phone many times when I send my message.

Q1.3: I get annoyed when I am kept waiting for response of my message.

Q1.4: I get worried if I don't receive messages for long time.

Q1.5: I feel lonely if I receive no message when I check my cell-phone.

Q1.6: I think a problem is happened in human relations if there is a human whose message response is slow.

Q1.7: I think a problem may happen in human relations if there are no exchange of message.

Table 3. Questionnaires relating to the participation attitude to the Internet for VAS (questions from Q2.1 to Q2.10)

Q2.1: I am always thinking about the Internet.

Q2.2: I get worried if I don't continue to use the Internet. Q2.3: I get worried if I receive no response to my message. Q2.4: I want to check my cell-phone many times when I send my message.

Q2.5: Although after midnight, I would continue to use the Internet for 1 hour or more.

Q2.6: My sleep time becomes shorter if my utilization time for the Internet is longer.

Q2.7: I use my cell-phone while walking or cycling.

Q2.8: I want to send a message or to use the Internet, although I converse with a person.

Q2.9: I'm good at communication via the Internet than face-to-face communication.

Q2.10: I want to avoid the face-to-face communication because my main communication is via the Internet. 


\section{Results and Discussion}

\subsection{Basic Statistics and Correlation Matrix}

Table 4 (a) shows the basic statistics of questionnaires relating to the sending and reception of messages based on VAS, from Q1.1 to Q1.7. All maximum values are 0.81 or more, otherwise, all minimum value is 0.00 . Therefore, distributions of these values are full range of these scales, and these are found to be large variation. However, other features are not clear.

On the other hand, Table 4 (b) shows the basic statistics from Q2.1 to Q2.10. From Table 4(b), distributions of Q2.1 to Q2.10 are regarded as full range of these scales, and these are found to be large variation too. And it is observed that medians of Q2.2, Q2.5, Q2.6 and Q2.10 are symmetric and characteristic. However, other features are not clear as same as Table 4 (a).

In addition, the correlation matrix of these question is shown in Table 5 (a). It is found that Q1.1 is moderately related to Q1.3 (r=0.62) and Q1.4 is also moderately related to $\mathrm{Q} 1.5(\mathrm{r}=0.79)$ from Table 5 (a). And it is found that other correlations are weak

Moreover, the correlation matrix from Q2.1 to Q2.10 is also shown in Table 5 (b). From Table 5 (b), Q2.1 is moderately related to $\mathrm{Q} 2.2(\mathrm{r}=0.76)$, and $\mathrm{Q} 2.9$ is also moderately related to $\mathrm{Q} 2.10(\mathrm{r}=0.70)$. And it is found that other correlations are weak as same as Table 5 (a).

That is, it is considered that relationships and meanings of subjective evaluation values or personalities of participants are not expressed from these components.

\subsection{Combination of Univariate Scatter Plots and Box-and-Whisker Plots}

To clarify relationships and meanings of Q1.1 to Q1.7, combination of univariate scatter plots and box-and-whisker plots ${ }^{(11)}$ are shown as Fig.3 (a). These distributions are found to be large variation, it can be observed that distribution of Q1.7's values is characteristic. Most of them are close to 0.00 , and all outliers of them are greater than 0.58 . This fact is the same with the results and discussion in Section 4.1 .

As same as Fig. 3 (a), to clarify relationships and meanings from Q2.1 to Q2.10, combination of univariate scatter plots and box-and-whisker plots ${ }^{(11)}$ are also shown in Fig.3 (b). Note that their features of distribution are different from Fig.3 (a)'s distribution.

However, these distributions are also found to be large
Table 4. Basic statistics

(a) Q1.1 to Q1.7

\begin{tabular}{|c|c|c|c|c|c|c|c|}
\hline & Q1.1 & Q1.2 & Q1.3 & Q1.4 & Q1.5 & Q1.6 & Q1.7 \\
\hline Max. & 0.88 & 0.85 & 0.78 & 0.98 & 0.98 & 0.87 & 0.81 \\
\hline Min. & 0.00 & 0.00 & 0.00 & 0.00 & 0.00 & 0.00 & 0.00 \\
\hline Mean & 0.29 & 0.37 & 0.24 & 0.26 & 0.26 & 0.23 & 0.14 \\
\hline Median & 0.19 & 0.31 & 0.19 & 0.14 & 0.12 & 0.11 & 0.03 \\
\hline SD* & 0.29 & 0.27 & 0.25 & 0.30 & 0.29 & 0.29 & 0.26 \\
\hline
\end{tabular}

(b) Q2.1 to Q2.10

\begin{tabular}{|c|c|c|c|c|c|}
\hline & Q2.1 & Q2.2 & Q2.3 & Q2.4 & Q2.5 \\
\hline Max. & 0.67 & 0.94 & 0.88 & 0.81 & 1.00 \\
\hline Min. & 0.00 & 0.00 & 0.00 & 0.00 & 0.00 \\
\hline Mean & 0.21 & 0.17 & 0.29 & 0.28 & 0.55 \\
\hline Median & 0.14 & 0.05 & 0.12 & 0.11 & 0.75 \\
\hline SD* & 0.05 & 0.08 & 0.10 & 0.08 & 0.15 \\
\hline & Q2.6 & Q2.7 & Q2.8 & Q2.9 & Q2.10 \\
\hline Max. & 1.00 & 0.73 & 0.96 & 1.00 & 0.97 \\
\hline Min. & 0.02 & 0.00 & 0.00 & 0.00 & 0.00 \\
\hline Mean & 0.66 & 0.35 & 0.46 & 0.46 & 0.22 \\
\hline Median & 0.69 & 0.32 & 0.51 & 0.38 & 0.04 \\
\hline SD* & 0.08 & 0.08 & 0.09 & 0.15 & 0.12 \\
\hline
\end{tabular}

*Standard deviation

Table 5. Correlation matrix

(a) Q1.1 to Q1.7

\begin{tabular}{|l|l|l|l|l|l|l|}
\hline & Q1.1 & Q1.2 & Q1.3 & Q1.4 & Q1.5 & Q1.6 \\
\hline Q1.2 & 0.36 & & & & & \\
\hline Q1.3 & $\mathbf{0 . 6 2}$ & 0.39 & & & & \\
\hline Q1.4 & 0.35 & 0.15 & 0.27 & & & \\
\hline Q1.5 & 0.05 & 0.36 & 0.08 & $\mathbf{0 . 7 9}$ & & \\
\hline Q1.6 & 0.39 & 0.16 & 0.13 & 0.08 & 0.02 & \\
\hline Q1.7 & 0.27 & -0.31 & 0.34 & 0.26 & -0.12 & 0.41 \\
\hline
\end{tabular}

(b) Q2.1 to Q2.10

\begin{tabular}{|l|l|l|l|l|l|l|l|l|l|}
\hline & Q2.1 & Q2.2 & Q2.3 & Q2.4 & Q2.5 & Q2.6 & Q2.7 & Q2.8 & Q2.9 \\
\hline Q2.2 & 0.76 & & & & & & & & \\
\hline Q2.3 & 0.09 & 0.13 & & & & & & & \\
\hline Q2.4 & 0.25 & 0.01 & 0.43 & & & & & & \\
\hline Q2.5 & 0.06 & -0.34 & 0.02 & -0.19 & & & & & \\
\hline Q2.6 & 0.49 & 0.27 & 0.01 & 0.17 & 0.39 & & & & \\
\hline Q2.7 & -0.18 & -0.15 & 0.08 & 0.20 & 0.04 & 0.22 & & & \\
\hline Q2.8 & 0.27 & 0.26 & 0.22 & -0.18 & 0.41 & 0.43 & -0.07 & & \\
\hline Q2.9 & 0.11 & 0.20 & -0.01 & 0.40 & -0.60 & -0.07 & -0.29 & -0.12 & \\
\hline Q2.10 & 0.30 & 0.41 & -0.31 & 0.00 & -0.38 & -0.16 & -0.52 & 0.07 & 0.70 \\
\hline
\end{tabular}




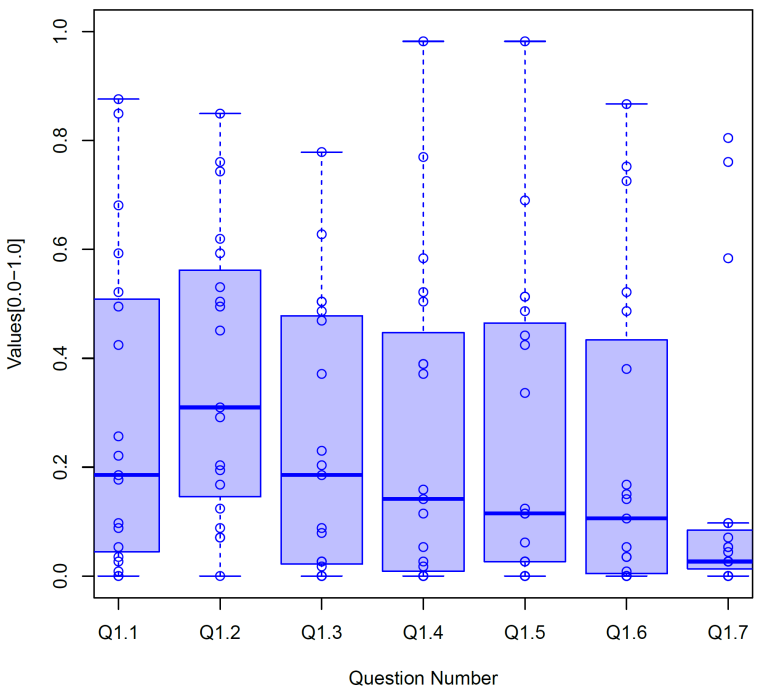

(a) Q1.1 to Q1.7

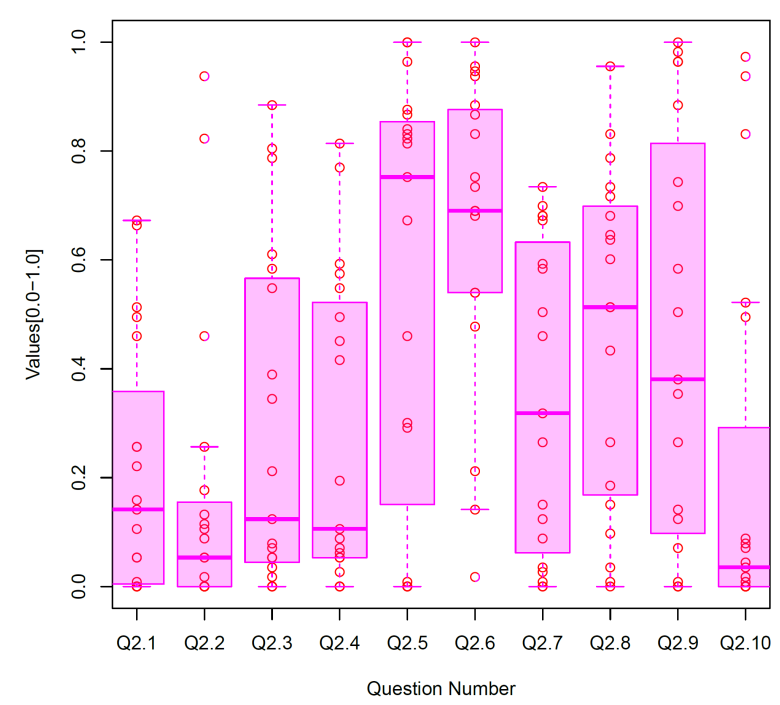

(b) Q2.1 to Q2.10

Fig.3. Combination of Univariate Scatter Plots and box-and-whisker plots

variation, and it is observed that distribution of Q2.2, Q2.5, Q2.6 and Q2.10 are characteristic. That is, all outliers of Q2.2 and Q2.10 are greater than 0.46, and their medians are lower than 0.05 . On the other hand, medians of Q2.5 and Q2.6 are over 0.69. These facts are evidences of the results and discussion of Section 4.1 .

In contrast, relationships and meanings of Q1.1 to Q1.7 and Q2.1 to Q2.10 are not clear. This observation is same as part of our previous studies ${ }^{(8-10)}$.

\subsection{Hierarchical Clustering Analysis by Ward} Method and Non-Hierarchical Clustering analysis by k-means

Under above results and discussion, to understand relationships and meanings of subjective evaluation values and clustering of participants as consistent with our feelings ${ }^{(8-10)}$, hierarchical clustering analysis by Ward method and non-hierarchical clustering analysis by k-means are carried out.

Fig. 4 shows the cluster dendrogram from Q1.1 to Q1.7. It is considered that these are divided into three clusters from Fig.3 (a) and Tsuruta et al.'s factor analysis ${ }^{(12)}$. Furthermore, the number of clusters is defined as 3, non-hierarchical clustering analysis by k-means are also carried out. This result is same as in Fig.4, clustering from Q1.1 to Q1.7 and their meanings ${ }^{(12)}$ are summarized in Table 6. In this way, relationships and meanings of subjective evaluation values of Q1.1 to Q1.7 are visualized and understood ${ }^{(8-10)}$.

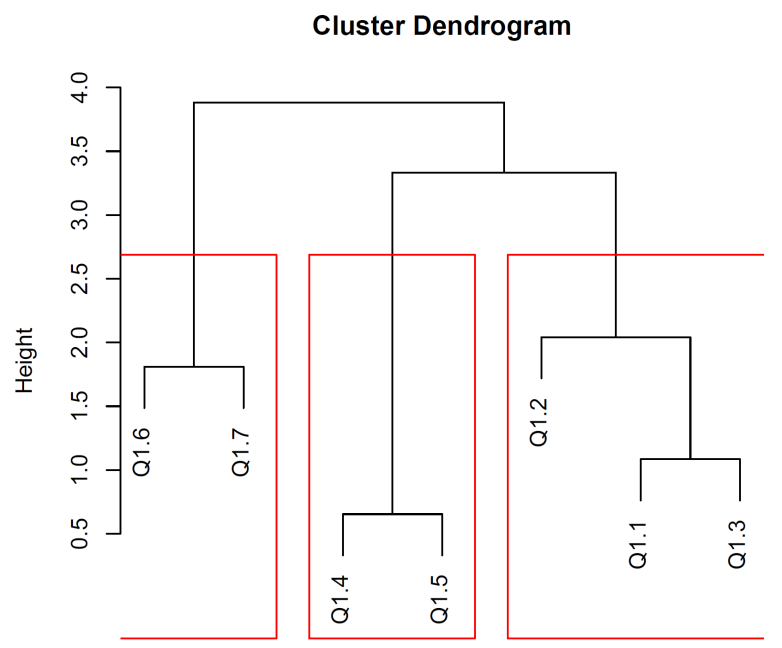

Question Number hclust (*, "ward.D2")

Fig.4. Cluster dendrogram from Q1.1 to Q1.7

Table 6. Clustering by k-means from Q1.1 to Q1.7 and their meanings

Cluster 1: Q1.4, Q1.5

Meaning: Question to seek sympathy for the Internet

Cluster 2: Q1.1, Q1.3, Q1.2

Meaning: Questions related to emotional stability for response of message

Cluster 3: Q1.6, Q1.7

Meaning: Questions about human relations 
And Fig. 5 shows the cluster dendrogram from Q2.1 to Q2.10. It is considered that these are divided into two clusters from Fig.3 (b) and Tsuruta et al.'s factor analysis ${ }^{(12)}$. Moreover, the number of clusters is defined as 2, non-hierarchical clustering analysis by k-means are also carried out. This result is same as in Fig.5, clustering from Q2.1 to Q2.10 and their meanings ${ }^{(12)}$ are summarized in Table 7. As same as Fig.4 and Table 6, relationships and meanings of subjective evaluation values of Q2.1 to Q2.10 are also visualized and understood ${ }^{(8-10)}$.

As mentioned above, relationships and meanings of subjective evaluation values are visualized and analyzed. This fact is consistent with our senses and it is evidence that can be analyzed by a small number of subjective evaluation measurement based on $\operatorname{VAS}^{(8-10)}$.

\section{Conclusions}

To analyze the relationships and meanings of subjective evaluation, this work carried out the visualizations of subjective evaluation for the participation

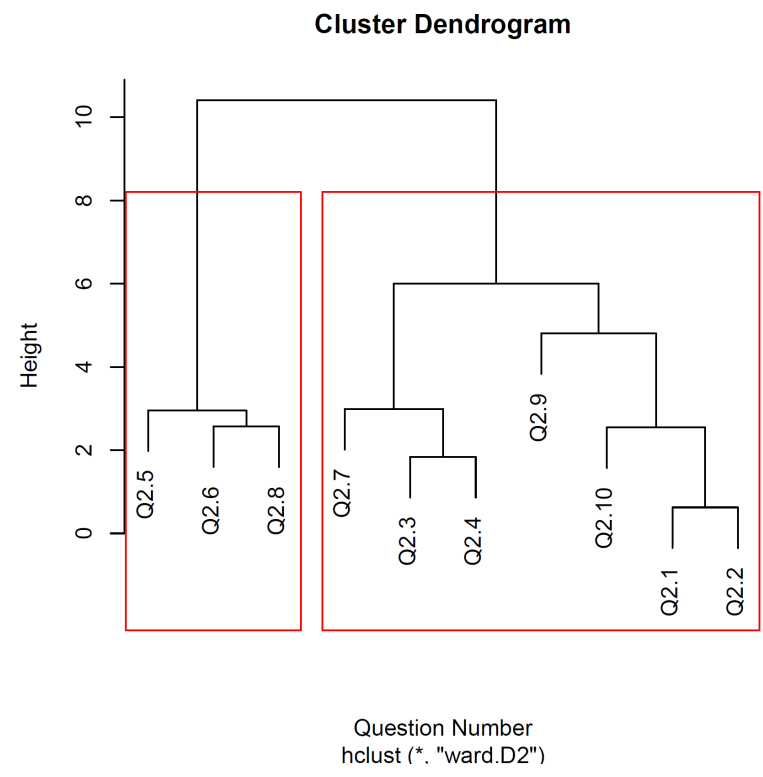

Fig.5. Cluster dendrogram from Q2.1 to Q2.10

Table 7. Clustering by k-means from Q2.1 to Q2.10 and their meanings

Cluster 1: Q2.1, Q2.2, Q2.10, Q2.9, Q2.3, Q2.4, Q2.7

Meaning: Utilization, attitude and communication for the Internet

Cluster 2: Q2.6, Q2.8, Q2.5

Meaning: Priority of utilization for the Internet attitude to the Internet of male college students by a small number of subjective evaluation measurement based on VAS. This method consists of following three procedures: calculation of basic statistics and a correlation matrix; drawing of combination of univariate scatter plots and box-and-whisker plots ${ }^{(11)}$; and hierarchical clustering analysis by Ward method and non-hierarchical clustering analysis by $\mathrm{k}$-means.

Under these procedures, the relationships and meanings of subjective evaluation are visualized and understood, they are consistent with our feelings and published article $\mathrm{e}^{(8-10,12)}$.

By the way, in general, it is necessary to use large measurements of subjective evaluation to obtain reliable conclusions. On the other hand, this proposed method is very useful to analyze by a small number of subjective evaluation measurement based on VAS.

To obtain reliable conclusions, applying this method in the future study and conducting more investigation are very important.

Future works are as follows: (1) clustering of participants (2) measurement with larger number of participants, (3) investigation of differences in LS measurement and VAS measurement, and (4) applying this method in another fields.

\section{Acknowledgment}

This work is partially supported by JSPS Grants-in-Aid for Scientific Research, No.16K01890.

\section{References}

(1) Likert, R. A.: Technique for the Measurement of Attitudes, Archives of Psychology (New York), 1932.

(2) Peabody, D.: Two components in bipolar scales: Direction and extremeness, Psychological Review, vol.69, No.2, pp.65-73, 1962.

(3) Upshaw, H.S.: Own attitude as an anchor in equal-appearing intervals, The Journal of Abnormal and Social Psychology, Vol.64, No.2, pp.85-96, 1962.

(4) Cummins, R. A.: The second approximation to an international standard for life satisfaction. Social Indicators Research, Vol.43 No.3, pp.307-334, 1998.

(5) Heine, S. J., Lehman, D. R, Peng, K. and Greenholtz, J.: What's wrong with cross-cultural comparisons of subjective Likert scales?: The reference-group effect, Journal of personality and social psychology, Vol.82, 
No.6, pp.903-918, 2002.

(6) Watanabe, S., Shirahama, N., Tsukamoto, H., Matsumoto, Y., Nakagawa, M., Miyamoto, K., Nakaya, N., Tomita, M. and Mori Y.:Trend of the Subjective Evaluation based on Visual Analog Scale and Likert Scale, ICIC Express Letters, Part B: Applications (ICIC-ELB), Vol. 7, No.1, p.37-42, 2016.

(7) Watanabe, S., Shirahama, N., Matsumoto, Y., Tsukamoto, H., Nakagawa, M., Miyamoto, K., Nakaya, N., Tomita, M. and Mori, Y.: Distribution Trend of the Information Skills for Students Based on Visual Analog Scale and Likert Scale, International Journal of Biomedical Soft Computing and Human Sciences, Vol. 20, No.2, pp.1-6, 2016.

(8) Watanabe, S., Shirahama, N., Matsumoto, Y., Tsukamoto, H., Nakaya, N. and Mori, Y.: Subjective Evaluation for the Impression of Music Based on Visual Analog Scale and Cluster Analysis, Journal of Biomedical Fuzzy Systems Association, Vol.19, No.2, pp.53-65, 2017 (in Japanese).

(9) Watanabe, S., Shirahama, N., Matsumoto, Y., Tsukamoto, H., Nakaya, N. and Mori, Y.: Proposal of a New Concept of Subjective Evaluation Measurement Based on Visual Analog Scale, Proceedings of the 30th Annual Conference of Biomedical Fuzzy Systems Association, pp.157-160, November 2017, Neyagawa, Japan (in Japanese).

(10) Shirahama, N., Watanabe, S., Ikegami, F., Nakaya N. and Mori, Y.: A Study on the Impression Received from the Response of a Communication Robot, Proceedings of the 5th IIAE International Conference on Intelligent Systems and Image Processing 2017 (ICISIP2017), pp.356-363, September 2017, Honolulu, Hawaii, U.S.A.

(11) Weissgerber, T. L., Milic, N. M., Winham, S. J., Garovic, V. D.: Beyond Bar and Line Graphs: Time for a New Data Presentation Paradigm. PLOS Biology, Vol.13, No.4: e1002128. doi:10.1371/journal.pbio.1002128, 2015.

(12) Tsuruta, T., Yamamoto, Y. and Nojima, E.: Development of the Internet Addiction Tendency Scale for High-School Students, Japan Journal of Educational Technology, Vol.37, No.4, pp.491-504, 2014 (in Japanese). 